\title{
ÉTUDE DES POPULATIONS \\ D'AUSTROPOTAMOBIUS PALLIPES (CRUSTACEA, ASTACIDAE) DANS UN RUISSEAU FORESTIER DE NORMANDIE. II. RÉPARTITION EN FONCTION DES HABITATS : STABILITÉ ET VARIABILITÉ AU COURS DE CINQ ANNÉES.
}

\author{
A. NEVEU
}

INRA, Laboratoire d'Écologie Aquatique, UMR EQHC, INRA-ENSAR, 65, rue de St Brieuc, 35042 Rennes Cedex.

\section{RÉSUMÉ}

Les populations d'Austropotamobius pallipes ont été étudiées dans deux sites, en aval et en amont, d'un ruisseau forestier. de Normandie au cours de cinq années (1991-1995). Au début d'octobre de chaque année les échantillons ont été prélevés à l'aide de filets à mailles fines $(3 \mathrm{~mm})$ au niveau des principales séquences de faciès dans les deux sites. Les séquences du site aval sont homogènes avec des graviers, des cailloux et quelques grosses pierres, en zones de courant peu profondes. Les séquences du site amont sont plus hétérogènes, certaines sont lotiques avec des graviers, des cailloux et de rares pierres sur lesquelles peuvent pousser des touffes de Fontinalis, d'autres sont lentiques avec du sable, du limon, de la vase et des amas de détritus organiques.

En aval les densités d'écrevisse sont assez comparables d'une séquence à l'autre, malgré une variabilité interannuelle significative. En amont les densités sont très variables dans l'espace et dans le temps, en liaison avec l'hétérogénéité des substrats. Une certaine ségrégation spatiale entre les classes de taille est visible en amont contrairement à l'aval. La répartition des adultes les plus grands est relativement homogène comparativement aux plus petits individus.

Le sex-ratio est stable dans les différentes séquences et les variations annuelles sont faibles.

L'ordre de préférence des séquences par les écrevisses adultes reste stable au cours des années, indépendamment des variations de densité. La sélection par les juvéniles de l'année est plus variable.

L'évolution de la densité des écrevisses en aval est indépendante de celle de l'amont. La densité des juvéniles est corrélée à la surface recouverte de cailloux et à la quantité de grosses pierres. Les adultes sont plus ubiquistes dans leur choix et leur densité est moins fortement liée à celle des pierres. 
Des changements accidentels dans la structure du substrat, comme l'accumulation de branches, de feuilles mortes, peuvent augmenter la population grâce à des abris plus nombreux. Mais l'accumulation de sédiments à l'amont de l'obstacle est défavorable aux écrevisses, en particulier pour les plus petites. De même les activités d'exploitation forestière augmentent la quantité de dépôts : il en résulte une réduction des effectifs des populations, en particulier dans le site amont de 1994 à 1995.

Cette étude montre que la densité des écrevisses est globalement stable au cours de la période considérée, malgré une certaine variabilité spatio-temporelle au niveau stationnel. Les différences de densité entre l'amont et aval peuvent aussi expliquer partiellement les différences de vitesse de croissance observées dans une précédente étude.

Tous ces résultats peuvent être utilisés pour l'aménagement du ruisseau de façon à améliorer l'habitat pour les écrevisses et à détecter les effets néfastes des activités forestières.

Mots-clés : Astacidae, Austropotamobius pallipes, microrépartition, structure de I'habitat, variabilité spatio-temporelle, ruisseau, Normandie, France.

\title{
STUDY OF AUSTROPOTAMOBIUS PALLIPES (CRUSTACEA, ASTACIDAE) POPULATIONS IN A FOREST BROOK IN NORMANDY. II. DISTRIBUTION IN RELATION TO HABITATS STRUCTURE : STABILITY AND VARIABILITY DURING FIVE YEARS.
}

\begin{abstract}
Austropotamobius pallipes populations were studied in two sites located downstream and upstream in a forest brook in Normandy (Western part of France) during five years (1991-1995). Every year and for each site, craytishes were sampled at the beginning of October month by hand nets with small mesh size $(3 \mathrm{~mm})$ in main facies sequences. Sequences in downstream site are homogeneous with gravels, pebbles and some boulders, shallow running waters. Sequences in upstream site are more heterogeneous, some of then are rheophilic with gravels, pebbles and scarce boulders with Fontinalis clumps, other ones are lennitic with sand, silt, mud and organic detritus.

In downstream site annual crayfish densities are quite similar whatever the sequence and annual variability is high. In upstream site crayfish densities change in space and time and are correlated to facies substrate heterogeneity. Spatial segregation between size classes is clearer upstream than downstream. Largest adults have a more homogeneous spreading between facies than smallest individuals.
\end{abstract}

Sex-ratio is stable between sequences and annual variations are weak.

In the two sites order of sequences chosen by crayfish adults is stable during years independently of density variations. Variability in choice is higher in the youngs of the year. 
Variations of crayfish density between downstream and upstream sites are independent. Density is correlated with pebbly area in the youngs of the year and with boulders quantity. Adults are more ubiquitous in their choice and their density less little correlated with boulders quantity.

Accidental variations in substratum structure, like packs of branches and of dead leaves, increase density by creating new shelters. But sand accumulation above this obstacle is unfavourable for crayfishes especially for smallest ones. Likewise forestry activities increase the quantity of deposed silt and consequently crayfish populations density is reduced in upstream site during 1994 and 1995.

This study shows that crayfish population density is quite stable during the study period in spite of local variability in space and time. Differences of density between both sites can also partly result in differences in growth rates compared to previous data.

All these results can be used to manage brook in order to improve favourable habitat for crayfishes and to detect deleterious effects of forestry activities.

Key-words : Astacidae, Austropotamobius pallipes, microdistribution, habitat structure, spacio temporal variability, brook, Normandy, France.

\section{INTRODUCTION}

L'écrevisse à pattes blanches Austropotamobius pallipes (Lereboullet, 1858) est en régression dans de nombreuses régions et de multiples hypothèses peuvent être élaborées quant aux causes. Elles peuvent être regroupées autour de deux idées principales : l'influence des pollutions et l'impact des pathologies. Dans le premier cas la dégradation de la qualité de l'eau est rarement distinguée de l'évolution de l'habitat, d'autant plus que les deux facteurs peuvent être liés. Pour comprendre cette évolution il est aussi nécessaire de faire appel à un état référentiel considéré comme l'habitat idéal pour l'espèce.

Certains auteurs ont essayé de définir le rôle de la qualité de l'eau et l'impact de sa dégradation sur les populations de cette écrevisse (BIKUNA et al., 1989 ; NAURA et ROBINSON, 1998). De même certaines études ont essayé de définir les habitats préférentiels (BROWN et BOWLER, 1977 ; DAGUERRE de HUREAUX et ROQUEPLO, 1981 ; LACHAT et LAURENT, 1988 ; FOSTER, 1995 ; BOHL, 1997). Mais peu d'observations portent sur le rôle fonctionnel de l'habitat, c'est-à-dire sur sa capacité à favoriser l'épanouissement des différentes phases du cycle biologique, à part celle de ROQUEPLO (1997) qui montre un certain choix dans la structure du substrat en fonction de l'âge des individus, ou encore celle de FOSTER (1993) qui trouve une relation entre la taille (donc l'âge) des individus et celle des abris accessibles. Or ces résultats sont importants dans la mesure où cette écrevisse est asséz casanière et retourne dans son faciès d'origine lorsqu'elle est déplacée (GHERARDI et al., 1998). Il est donc possible de s'interroger sur les relations entre cet animal et l'état structurel de son habitat selon sa taille, son âge, le sexe considéré, mais surtout sur la stabilité de ces relations dans le temps et l'espace, sur plusieurs années et sites.

Des travaux précédents montrent la richesse en écrevisses à pattes blanches des ruisseaux du Parc Naturel Régional Maine-Normandie (NEVEU, 1996). Mais ces populations sont fragiles, sanctuarisées dans des zones forestières où les conditions environnementales sont a priori tamponnées par la présence de la futaie. Elles peuvent cependant disparaître, ce fut le cas sur le bassin de la Cance au cours de l'été particulièrement chaud de 1990 (NEVEU, op. cit.). 
Aussi une étude sur plusieurs années a alors été entreprise pour savoir si cet environnement forestier était garant de la protection de l'espèce. Les premiers résultats ont porté sur la croissance et sa variabilité au cours de 6 années, dans deux sites d'un petit ruisseau : le ruisseau du Vivier (NEVEU, 2000). Dans ce biotope des analyses ont aussi été effectuées sur la microdistribution des individus en fonction des différents faciès, sur l'incidence de la taille des individus ou du sexe sur le choix d'un substrat, mais surtout sur l'évolution temporelle des populations en relation avec la stabilité et/ou la variabilité des habitats.

Dans cette étude la notion d'habitat est employée à l'échelle du " mésohabitat ", c'est-à-dire à un niveau " moyen » de mesure (quelques mètres), ce qui correspond à une bonne approche fonctionnelle de l'écosystème en vue de sa gestion (ORTH, 1995). Ce niveau d'échelle correspond aussi à la couverture de deux besoins essentiels : la sécurité (abri : repos, protection contre la lumière, protection anti prédateur) et l'alimentation. Cette zone est classiquement considérée comme la "zone d'activité " de l'animal, sensu LEVEQUE (1995). Ce qui se justifie d'autant plus que les déplacements trophiques de cette écrevisse sont limités (quelques mètres au plus), sont nocturnes et que très peu d'animaux quittent cette zone au cours de l'année (GHERARDI et al., 1998).

\section{MÉTHODES D'ÉTUDE}

\section{Localisation des sites}

Le ruisseau du Vivier ( $0^{\circ} 31^{\prime} 00^{\prime \prime}$ Ouest, 48 $35^{\prime} 32^{\prime \prime}$ Nord) est un affluent d'ordre 2 de l'Andainette qui coule dans la région de la ville de Domfront en Normandie (partie ouest de la France). Ce ruisseau est issu de 2 étangs forestiers, sa longueur totale est de $3,4 \mathrm{~km}$, sa largeur de 1 à $3 \mathrm{~m}$. Le site d'étude aval (A) est à $100 \mathrm{~m}$ de la confluence avec l'Andainette, le site amont (B) est à $1,8 \mathrm{~km}$, soit à $1,5 \mathrm{~km}$ des étangs (NEVEU, 2000). La structure géologique dominante est constituée par des grès.

Dans chaque site la zone étudiée peut être subdivisée en parties relativement homogènes correspondant à un certain type de mésohabitat. Chacune de ces parties peut être considérée comme une " séquence " sensu MALAVOI (1989) et peut comporter de deux à trois types de faciès. Le faciès étant défini par l'auteur précédent comme une zone où la pente, la vitesse et la profondeur sont comparables. Dans l'ensemble étudié 8 faciès de base peuvent être définis dont 6 principaux à savoir :

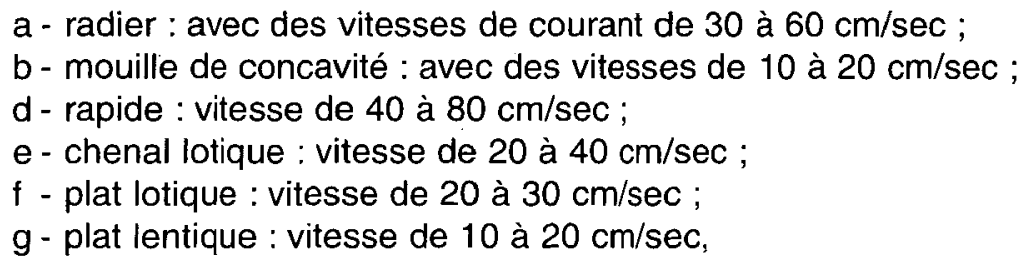

et 2 faciès accessoires:

c - banc de convexité : vitesse de 0 à $20 \mathrm{~cm} / \mathrm{sec}$;

$\mathrm{h}$ - fosse de dissipation : vitesse de 10 à $30 \mathrm{~cm} / \mathrm{sec}$, très variable. Faciès très imbriqué avec le suivant, souvent une mouille de concavité.

Les vitesses de courant ont été estimées à partir de mesures ponctuelles effectuées dans les différents faciès à l'aide d'un micromoulinet. 
Le site A présente une largeur régulière proche de $2 \mathrm{~m}$, la zone étudiée mesure $27 \mathrm{~m}$ de longueur, la pente est de $20 \%$, ce qui se traduit par un habitat lotique relativement régulier et un substrat à cailloux dominants. Ce site se trouve sous un couvert forestier homogène constitué par un taillis de feuillus. La zone d'étude peut être subdivisée en 3 séquences homogènes, d'égale surface $\left(18 \mathrm{~m}^{2}\right)$ (Figure 1$)$ :

A1 : 3 faciès. 2 faciès principaux : mouille de concavité, radier, 1 faciès accessoire : fosse de dissipation. La partie la plus en aval est la plus riche en cailloux ( $73 \%$ de la surface, 4,4 grosses pierres de plus de $20 \mathrm{~cm}$ par $\mathrm{m}^{2}$ ), les éléments fins sont absents (Tableau I). La profondeur est faible (15 à $20 \mathrm{~cm}$ ), plus accentuée, à l'amont avec une fosse caillouteuse de $40 \mathrm{~cm}$ de profondeur, bordée par des racines d'aulnes et avec une berge légèrement creusée.

A2 : 3 faciès. 2 faciès principaux : chenal lotique, plat-radier, 1 faciès accessoire : rapide. Zone caillouteuse régulière, de faible profondeur $(20 \mathrm{~cm})$, légèrement plus profonde au centre le long d'une berge faiblement creusée, avec quelques éléments fins (17\% de la surface).

A3 : 3 faciès. 2 faciès principaux : chenal lotique, plat-radier, 1 faciès accessoire : fosse de dissipation. Les graviers deviennent dominants (56\% de la surface), la bathymétrie est très contrastée, une plage de graviers borde un profond où certains éléments fins se déposent. Les cailloux sont limités à la zone de courant qui est bordée par une berge légèrement creusée et quelques racines d'aulnes.

En amont le site $B$ présente un aspect très différent et contrasté. II se caractérise par une pente plus faible (5\%) une largeur plus variable et des faciès divers composés en grande partie de sédiments fins associés à un courant modéré. Ce site est plus éclairé en relation avec des feuillus âgés (chênes), un sous bois clairsemé sur la rive droite, la rive gauche étant couverte par un boisement d'épicéa, mais qui ne surplombe pas le ruisseau. L'éclairement du site, surtout dans sa partie médiane est nettement plus élevé qu'en aval. Des sous secteurs peuvent être définis à partir des limites de diverses séquences, chaque surface étant différente (Figure 2).

B4 : 2 faciès. 1 faciès plat lotique, 1 faciès plat lentique. Zone de courant modéré avec quelques cailloux reposant sur des graviers et du sable, les morceaux de branches sont fréquents. Ce secteur est demi ombragé par quelques aulnes.

B5 : 2 faciès. 1 faciès accessoire : plat lotique, 1 faciès principal : mouille de concavité sablo limoneuse avec de rares cailloux et une zone d'accumulation de feuilles mortes plus ou moins envasées.

B6 : 3 faciès. 1 faciès principal : radier, 2 faciès accessoires : plat-radier, banc de convexité. Cette séquence est constituée de cailloux, de graviers et d'un peu de sable en bordure. La berge est creusée au maximum de la concavité. Les cailloux sont souvent colonisés par des touffes de Fontinalis $s p$.

B7 : 4 faciès. 2 faciès principaux : mouille de concavité, plat (amont), 2 faciès accessoires : plat (aval), banc de convexité. Cet ensemble présente un point commun : à savoir des sédiments fins dominants. C'est une zone de dépôts (sédiments, feuilles mortes, débris), avec un profond central et une berge légèrement creusée, les cailloux sont rares, présents dans la partie amont avec quelques branches. Le banc de convexité est la partie la plus limoneuse avec des débris et des feuilles mortes plus ou moins enfouis.

Ces deux dernières séquences sont les plus éclairées et reçoivent du soleil au moins une partie de la journée. 


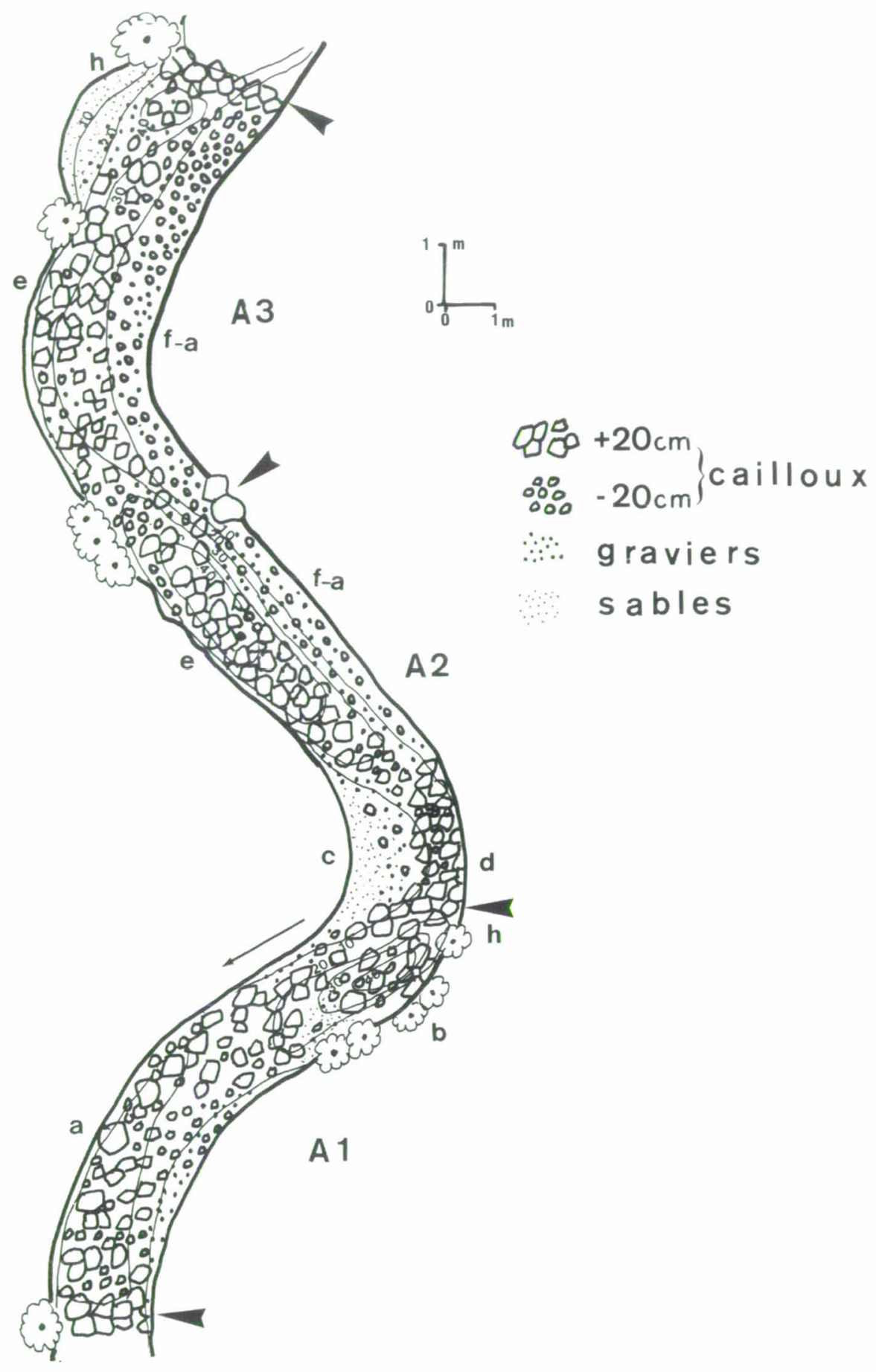

\section{Figure 1}

Localisation des principaux substrats et faciès du site aval $A$ (équidistances des lignes bathymétriques : $10 \mathrm{~cm}$. a : radier, $b$ : mouille de concavité, $c:$ banc de convexité, $d$ : rapide, $e$ : chenal lotique, $f:$ plat lotique, $h$ : fosse de dissipation).

\section{Figure 1}

Localization of main substrata and facies of downstream site $A$ (bathymetric lines equidistance : $10 \mathrm{~cm}$. a : riffle, b : pool, c : point bar, d : rapids, e : lotic channel, $f$ : run, $h$ : plunge pool). 


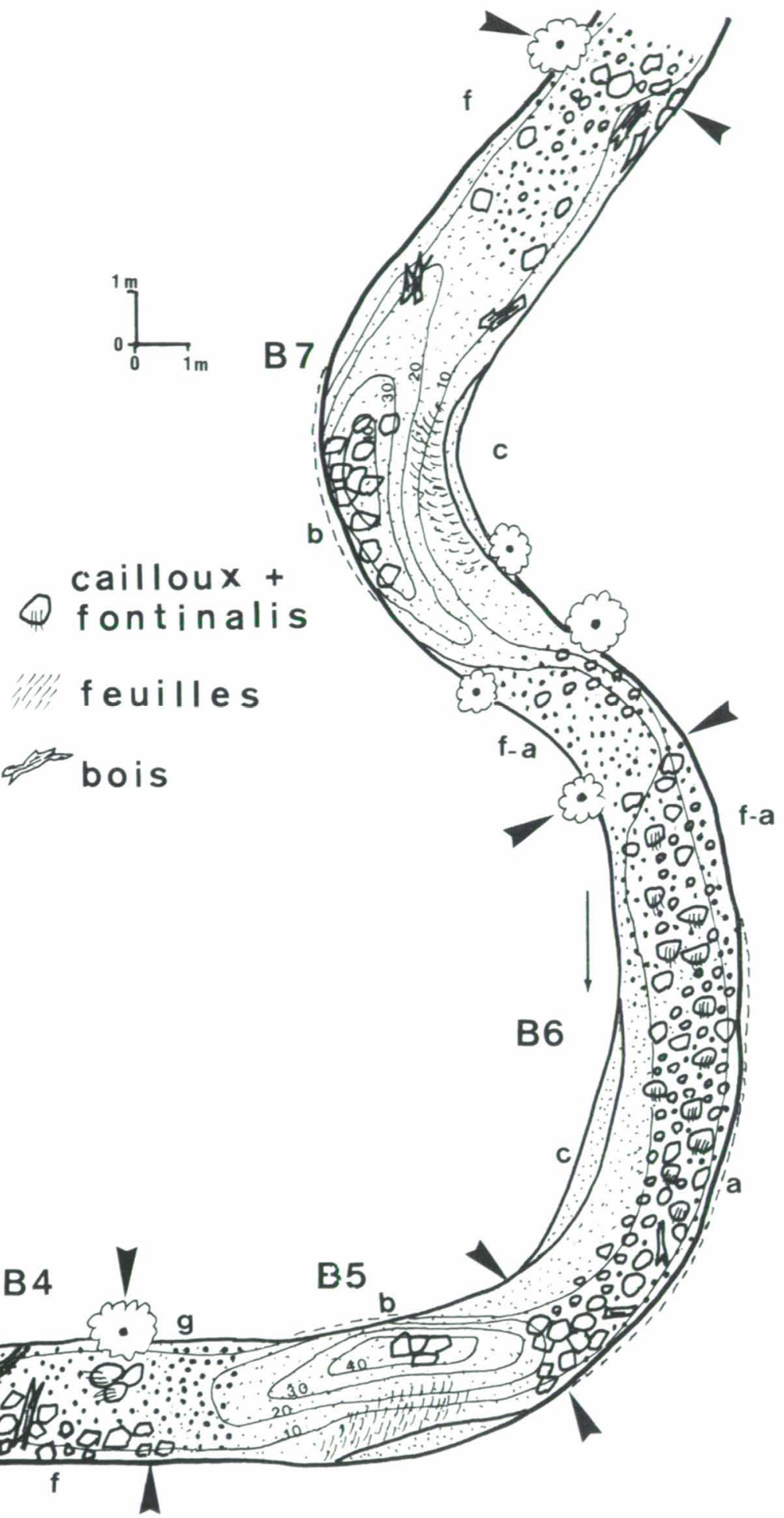

Figure 2

Localisation des principaux substrats et faciès du site amont B. (a : radier, b : mouille de concavité, $c$ : banc de convexité, $f$ : plat lotique, $g$ : plat lentique).

\section{Figure 2}

Localization of main substrata and facies of upstream site B. (a : riffle, b : pool, $c$ : point bar, $f$ : run, $g$ : flat). 


\section{Tableau I}

Principales caractéristiques des séquences des sites d'étude.

\section{Table I}

Main characteristics of study sites sequences.

\begin{tabular}{|c|c|c|c|c|c|c|c|}
\hline \multicolumn{7}{|l|}{} & \multicolumn{6}{|c|}{ SUBSTRATS } \\
\hline Faciès & Surface $\mathbf{m}^{2}$ & Cailloux $\%$ & Graviers $\%$ & Sables \% & Détritus \% & $\begin{array}{c}\text { Cailloux }+20 \mathrm{~cm} \\
\mathbf{N} / \mathbf{m}^{2}\end{array}$ \\
\hline Aval & \multicolumn{7}{|c|}{} \\
\hline A1 & 18 & 73 & 27 & 0 & 0 & 4,4 \\
\hline A2 & 18 & 56 & 27 & 17 & 0 & 3,3 \\
\hline A3 & 18 & 28 & 56 & 16 & 0 & 2,7 \\
\hline Amont & & & & & & \\
\hline B4 & 9 & 22 & 44 & 33 & 0 & 1,3 \\
\hline B5 & 16 & 7 & 0 & 81 & 12 & 0,2 \\
\hline B6 & 25 & 32 & 56 & 12 & 0 & 2,0 \\
\hline B7 & 40 & 15 & 30 & 45 & 10 & 1,0 \\
\hline
\end{tabular}

\section{Techniques de capture}

Les échantillons ont été capturés à l'aide d'épuisettes à mailles de $3 \mathrm{~mm}$, en remuant le substrat pour déloger les animaux selon la technique exposée par NEVEU (2000). Cette technique permet une localisation spatiale précise des écrevisses et leur remise en place dans leur habitat d'origine.

\section{Méthodes d'analyse}

Au niveau de la nomenclature, tous les animaux de l'année en cours, c'est-à-dire nés en juin-juillet et capturés en octobre, sont dénommés « juvéniles ", leur taille étant inférieure à $20 \mathrm{~mm}$. Tous les autres individus sont dénommés " individus $\geq 1+$ ", ils peuvent se décomposer en immatures $(20-40 \mathrm{~mm})$, subadultes $(41-60 \mathrm{~mm})$ et adultes $(+60 \mathrm{~mm})$.

Les échantillons ont été prélevés au début de chaque mois d'octobre de 1991 à 1995 en essayant de maintenir une pression de pêche régulière sous la forme de 2 passages sur chaque site durant environ 2 heures. Les pêches ont été effectuées de jour, période où les écrevisses sont en majorité dans leurs abris. L'efficacité de ces captures est pour les juvéniles de $27 \%$ en aval, $29 \%$ en amont, pour les adultes respectivement de 42 et $65 \%$. Ces valeurs sont estimées à partir de la méthode capture-marquage-recapture qui permet d'évaluer la densité la plus probable de la population. Les efficacités moyennes ont été calculées sur un ensemble de 5 inventaires par site (2 pêches successives), d'une manière indépendante pour les juvéniles (1987 individus contrôlés) et les animaux plus âgés (714 individus contrôlés). L'efficacité est estimée par le rapport des animaux indépendants (somme des captures de 2 pêches successives moins les marqués recapturés de la $2^{\text {ème }}$ pêche) à l'estimation de la population la plus probable (données non publiées). L'efficacité des pêches est plus élevée en amont pour les individus $\geq 1+$ en relation avec un substrat plus fin, moins d'abris, un courant modéré. Aussi pour la comparaison des deux sites, celui en aval nécessite l'emploi d'un 
facteur correctif de 1,56 de façon à se placer à un niveau comparable à l'amont quant à la représentativité des captures. Par contre dans la mesure où les efficacités des captures de juvéniles sont proches, l'analyse sur ces animaux peut se faire directement sur les deux sites, mais indépendamment de celle des adultes en rapport avec des efficacités plus faibles que sur ces derniers.

L'approche statistique est effectuée à partir des tests classiques du chi carré $\left(\chi^{2}\right)$, du coefficient de corrélation de rang de Spearman (Rs) dans les conditions exposées par SCHERRER (1984) au seuil de 5 pour cent.

\section{RÉSULTATS}

\section{Relation entre la densité des captures et le mésohabitat}

Compte tenu des différences de séquences entre l'amont et l'aval, en particulier au niveau de la structure des habitats, il est préférable d'analyser séparément les deux sites.

\section{Site aval (A)}

Les trois séquences ont une surface semblable, il est donc possible d'analyser directement les résultats intrasite entre eux.

La comparaison des captures d'individus $\geq 1+$ pour chaque année montre une hétérogénéité interséquences en 1991 et $1995\left(\chi^{2}=15,9\right.$ et 8,1$)$ contrairement aux autres années (Tableau II).

\section{Tableau II}

Evolution des captures sur le site aval en fonction des années et des séquences ( $N$ : nombre individus $\geq 1+$, Juv : nombre de juvéniles, $m$ : moyenne annuelle par séquence de $18 \mathrm{~m}^{2}, \sigma$ : écart type, cv : coefficient de variation, valeurs limites de $\chi^{2}: 5,9$ et 9,5$)$.

\section{Table II}

Catches evolution in downstream site in relation to years and sequences $(N$ : individuals $\geq 1+$ number, Juv : youngs of the year number, $m$ : annual average per sequence of $18 \mathrm{~m}^{2}, \sigma$ : standard deviation, $\mathrm{cv}$ : variation coefficient, threshold values of $\chi^{2}: 5.9$ and 9.5$)$.

\begin{tabular}{|c|c|c|c|c|c|c|c|c|c|c|}
\hline \multirow{2}{*}{} & \multicolumn{2}{|c|}{ A1 } & \multicolumn{2}{c|}{ A2 } & \multicolumn{2}{c|}{ A3 } & \multicolumn{2}{c|}{ TOTAL } & \multicolumn{2}{c|}{$\chi^{2}(5,9)$} \\
\cline { 2 - 13 } & $\mathbf{N}$ & Juv & $\mathbf{N}$ & Juv & $\mathbf{N}$ & Juv & $\mathbf{N}$ & Juv & $\mathbf{N}$ & Juv \\
\hline 1991 & 66 & 18 & 68 & 29 & 111 & 22 & 245 & 69 & $15,9^{\star}$ & 2,7 \\
\hline 1992 & 81 & 64 & 63 & 25 & 66 & $16:$ & 210 & 105 & 2,6 & $37,7^{\star}$ \\
\hline 1993 & 76 & 44 & 54 & 22 & 59 & 14 & 189 & 80 & 4,2 & $18,1^{\star}$ \\
\hline 1994 & 93 & 57 & 73 & 46 & 72 & 22 & 238 & 125 & 3,5 & $15,3^{\star}$ \\
\hline 1995 & 105 & 19 & 119 & 22 & 79 & 12 & 303 & 53 & $8,1^{\star}$ & 2,9 \\
\hline Total & 421 & 192 & 377 & 144 & 387 & 96 & 1185 & 432 & 2,6 & 38,2 \\
\hline$\chi^{2}(9,5)$ & 10,9 & $44,6^{\star}$ & $33,8^{\star}$ & $13,9^{\star}$ & $21,1^{\star}$ & 4,9 & $31,4^{\star}$ & $24,1^{\star}$ & - & - \\
\hline $\mathrm{m}$ & 84,2 & 40,4 & 75,4 & 28,8 & 77,4 & 17,2 & 237 & 86,4 & - & - \\
\hline$\sigma$ & 15,5 & 21,2 & 25,3 & 10,0 & 20,1 & 4,6 & 43,1 & 28,7 & - & - \\
\hline $\mathrm{cv}$ & 0,18 & 0,52 & 0,33 & 0,35 & 0,26 & 0,26 & 0,68 & 0,33 & - & - \\
& & & & & & & & & & \\
\hline
\end{tabular}


La comparaison des captures dans chaque séquence montre une hétérogénéité interannuelle $\left(\chi^{2}=10,9\right.$ pour $A 1, \chi^{2}=33,8$ pour $A 2, \chi^{2}=21,1$ pour $\left.A 3\right)$, avec globalement un maximum d'individus en 1995 et un minimum en 1992-1993.

En considérant l'ensemble des données comme une moyenne caractéristique du site, il n'y a pas de différence de densité entre les 3 séquences $\left(\chi^{2}=2,6\right)$, mais seulement entre les années $\left(\chi^{2}=31,4\right)$.

La comparaison des captures de juvéniles dans les différentes séquences et pour chaque année montre une hétérogénéité en 1992, 1993 et 1994 ; c'est-à-dire l'inverse des résultats pour les individus $\geq 1+$.

Les résultats pour les juvéniles, sur l'ensemble des captures, montrent une différence entre les séquences $\left(\chi^{2}=24,1\right)$, avec une réduction progressive des captures de l'aval vers l'amont du site. Cette réduction est régulière $(\mathrm{N}=-11,6 \mathrm{Ai}+52, \mathrm{n}=3, \mathrm{r}=1$, Ai étant le numéro de séquence et $\mathrm{N}$ le nombre de juvéniles pour une année moyenne). La comparaison des captures totales pour chacune des années montre une différence interannuelle $\left(\chi^{2}=38,2\right)$. L'analyse au niveau de chaque séquence indique que A2 et A3 évoluent de la même façon (Rs $=0,98$, valeur limite 0,90$)$ malgré des densités différentes. La variabilité des captures est plus élevée en A1 (coefficient de variation $52 \%$ contre $26 \%$ en $\mathrm{A3}$ ).

\section{Site amont $(B)$}

La comparaison interséquences nécessite une pondération des résultats bruts en relation avec des surfaces d'échantillonnage différentes (Tableau I), toutes les données sont ainsi rapportées à $10 \mathrm{~m}^{2}$ (Tableau III).

\section{Tableau III}

Evolution des captures sur le site amont en fonction des années et des séquences ( $N$ : nombre individus $\geq 1+$, Juv : nombre de juvéniles, $d$ : moyenne annuelle des densités de capture pour $10 \mathrm{~m}^{2}$ pour chaque séquence, $\sigma$ : écart type, cv : coefficient de variation, valeurs limites de $\chi^{2}: 7,8$ et 9,5 ).

\section{Table III}

Catches evolution in upstream site in relation to years and sequences ( $N$ : individuals $\geq 1+$ number, Juv : youngs of the year number, $d$ : annual average of catch density per $10 \mathrm{~m}^{2}$ for each sequence, $\sigma$ : standard deviation, $\mathrm{cv}$ : variation coefficient, threshold values of $\chi^{2}: 7.8$ and 9.5).

\begin{tabular}{|c|c|c|c|c|c|c|c|c|c|c|c|c|}
\hline \multirow{2}{*}{} & \multicolumn{2}{|c|}{ B4 } & \multicolumn{2}{c|}{ B5 } & \multicolumn{2}{c|}{ B6 } & \multicolumn{2}{c|}{ B7 } & \multicolumn{2}{c|}{ Site B } & \multicolumn{2}{c|}{$\chi^{2}(7,8)$} \\
\cline { 2 - 14 } & N & Juv & N & Juv & N & Juv & N & Juv & N & Juv & N & Juv \\
\hline 1991 & 15,5 & 1,1 & 8,1 & 0 & 28,8 & 0,8 & 9,0 & 0 & 15,3 & 0,4 & $17,8^{\star}$ & 2 \\
\hline 1992 & 6,6 & 3,3 & 0,6 & 3,7 & 24,4 & 23,6 & 4,7 & 3,2 & 9,1 & 8,4 & $36,5^{\star}$ & $36,2^{\star}$ \\
\hline 1993 & 10 & 26,6 & 7,5 & 10,6 & 37,6 & 26,4 & 11,5 & 11,2 & 16,6 & 18,7 & $35,6^{\star}$ & $13,0^{\star}$ \\
\hline 1994 & 11,1 & 0 & 9,3 & 1,2 & 32 & 4,4 & 12,2 & 2,5 & 16,1 & 2,0 & $20,9^{\star}$ & 5,2 \\
\hline 1995 & 15,5 & 3,3 & 6,2 & 1,8 & 17,6 & 5,6 & 5,5 & 2 & 11,2 & 3,1 & $10,4^{\star}$ & 2,8 \\
\hline Total & 58,7 & 34,3 & 31,7 & 17,3 & 140,4 & 60,8 & 42,9 & 18,9 & 68,3 & 32,6 & $80,1^{*}$ & $37,1^{\star}$ \\
\hline$d$ & 11,7 & 6,8 & 6,3 & 3,4 & 28,1 & 12,1 & 8,6 & 3,7 & 13,9 & 6,3 & - & - \\
\hline$\sigma$ & 3,8 & 11,2 & 3,4 & 4,2 & 7,6 & 11,8 & 3,4 & 4,3 & 3,8 & 6,7 & - & - \\
\hline $\mathrm{Cv}$ & 0,32 & 1,62 & 0,53 & 1,21 & 0,27 & 0,97 & 0,39 & 1,14 & 0,28 & 1,05 & - & - \\
\hline$\chi^{2}$ & 4,9 & $73,7^{\star}$ & 7,3 & $20,7^{\star}$ & 8,2 & $46,1^{\star}$ & 5,3 & $19,9^{\star}$ & 4,1 & $28,5^{\star}$ & - & - \\
$(9,5)$ & & & & & & & & & & & & \\
\hline
\end{tabular}


Les captures totales ainsi pondérées montrent pour les individus $\geq 1+$, une très grande hétérogénéité: interséquences $\left(\chi^{2}=80,1\right)$. Par contre les variations interannuelles sont homogènes $(\chi \doteq 4,1)$. La comparaison des séquences deux à deux montre que pour l'ensemble des 5 années B4 donne des captures semblables à B7 $\left(\chi^{2}=2,4\right)$ et B5 à B7 $\left(\chi^{2}=1,6\right)$, par contre $B 4$ diffère de $B 5\left(\chi^{2}=8,1\right)$. La séquence $B 6$ est la plus originale $\left(\chi^{2}=34,2\right.$ avec $B 4,68,6$ avec $B 5,51,4$ avec B7). La variabilité annuelle est la plus forte en $\mathrm{B} 5(\mathrm{cv}=53 \%)$ en relation avec la densité basse et elle est la plus faible en B6 ( $c v=26,7 \%$ ), zone.la plus peuplée.

Pour les juvéniles les différences interséquences sur l'ensemble des captures est forte $\left(\chi^{2}=28,5\right)$, même si l'ensemble du site est colonisé par ce stade. La comparaison des séquences deux à deux, montre que les zones profondes et limoneuses B5 et B7 sont faiblement peuplées mais comparables $\left(\chi^{2}=0,07\right)$. La variabilité interannuelle est très forte, elle est maximum en B4 ( $c v=162 \%)$ et minimum en B6 zone la plus peuplée ( $c v=97 \%$ ), elle peut s'expliquer par la faible capturabilité.

\section{Relation entre le mésohabitat et le sex-ratio}

En aval la fréquence des mâles, calculée sur la moyenne annuelle des captures, est comparable dans chaque séquence $\left(\chi^{2}=2,3\right.$ ), (Tableau IV A) par contre ie sex-ratio change au cours des années sur l'ensemble du site $\left(\chi^{2}=27,9\right)$. Ces différences sont liées à des variations daṇs les séquences $A 2\left(\chi^{2}=18,8\right)$ et $A 3\left(\chi^{2}=11,6\right)$, contrairement à $A 1$ où le rapport des sexes reste stable $\left(\chi^{2}=4,5\right)$.

\section{Tableau IV}

Evolution du sex-ratio (fréquence des mâles) au cours des années dans les différentes séquences des sites $\mathbf{A}$ et $B$.

\section{Table IV}

Sex-ratio changes (males frequency) along years in the various sequences of sites $A$ and $B$.

\begin{tabular}{|c|c|c|c|c|c|}
\hline \multicolumn{6}{|l|}{ A - AVAL } \\
\hline & $\therefore A 1$ & A2 & $\overline{A 3}$ & Total & \\
\hline 1991 & 0,48 & 0,60 & 0,45 & 0,50 & \\
\hline 1992 & 0,47 & 0,47 & 0,36 & 0,43 & \\
\hline 1993 & 0,47 & 0,50 & 0,47 & 0,48 & \\
\hline 1994 & 0,46 & 0,49 & 0,48 & 0,47 & \\
\hline 1995 & $\because 0,47$ & 0,51 & 0,49 & 0,47 & \\
\hline Total & $: 0,47$ & 0,51 & 0,44 & 0,47 & \\
\hline \multicolumn{6}{|c|}{ B - AMONT } \\
\hline & B4 & $\ldots$ B5 & $B 6 \cdot$ & $\because \mathrm{B7}$ & Total \\
\hline 1991 & 0,42 & 0,30 & 0,42 & 0,50 & 0,42 \\
\hline 1992 & $. .0,33$ & - & $\therefore 0,44$ & 0,42 & 0,42 \\
\hline 1993 & $\because 0,33$ & $\therefore 0,50 \cdots$ & $\therefore \quad 0,42$ & $-0,41$ & 0,41 \\
\hline 1994 & $\therefore 0,50$ & 0,33 & 0,42 & 0,43 & 0,43 \\
\hline 1995 & $\therefore 0,35$ & 0,20 & 0,45 & 0,42 & 0,42 \\
\hline Total & 0,39 & $\because \quad 0,33$ & $\because \quad 0,42$. & 0,44 & 0,42 \\
\hline
\end{tabular}


En amont le sex-ratio sur l'ensemble des années ne diffère pas entre les séquences $\left(\chi^{2}=3,5\right)$, il ne varie pas d'une année à l'autre sur l'ensemble du site $\left(\chi^{2}=0,04\right)$ (Tableau IV B). Au niveau de chaque séquence il reste stable au cours des années $\left(\chi^{2}\right.$ entre 0,5 et 1,7$)$.

\section{Relation entre la taille des individus $\geq 1+$ et le mésohabitat}

Les écrevisses au-delà de $20 \mathrm{~mm}$ sont classées en trois grandes catégories : les immatures $(20-40 \mathrm{~mm})$, les subadultes $(41-60 \mathrm{~mm})$ et les adultes (supérieures à $60 \mathrm{~mm}$ ).

En aval les captures dans chaque catégorie sont significativement différentes quelle que soit la séquence $\left(\chi^{2}: A 1=85,5 ; A 2=43,9 ; A 3=46,5\right)$ ce qui est en relation avec la réduction de densités avec l'âge (Tableau V). Quant à l'influence des substrats sur la représentativité des différentes tailles au cours des années, il n'y a pas de différence entre séquences pour les tailles subadultes et adultes $\left(\chi^{2}: 1,1\right.$ et 1,3$)$. Par contre pour les immatures il y a des différences $\left(\chi^{2}=5,9\right)$, la séquence $A 1$ étant plus favorable. L'analyse plus précise au niveau de chaque année donne des résultats variables quant à une éventuelle sélection de substrat par une classe de taille (Tableau VI). Les différences de captures sont significatives pour les immatures en 1993 et 1995, pour les subadultes en 1991 et 1994, pour les adultes en 1991.

En amont les captures dans chaque catégorie de taille sont différentes sur l'ensemble du site $\left(\chi^{2}=122,2\right)$. II apparaît des différences entre les séquences, les captures des trois catégories de tailles sont semblables dans B4 et B7 (Tableau VII A). Les grandes écrevisses représentent respectivement, de l'aval vers l'amont, : $37,7 \%-39,2 \%-59,5 \%-45,9 \%$ des captures. Les séquences B6 et B7 sont donc a priori plus représentatives de la structure démographique réelle de la population.

La comparaison des densités pondérées et rapportées à $10 \mathrm{~m}^{2}$, montre une différence de peuplement pour les immatures $\left(\chi^{2}=19,2\right)$, mais pas pour les subadultes $\left(\chi^{2}=5,7\right)$ ni pour les adultes $\left(\chi^{2}=0,3\right)$ (Tableau VII B). Ces dernières présentent la répartition la plus équitable dans les différentes séquences avec un coefficient de variation faible (36\%). La comparaison plus précise des divers habitats entre eux montre que les densités sont faibles et comparables dans B4, B5 et B7 pour les immatures $\left(\chi^{2}=0,6\right)$, alors que $\mathrm{B} 6$ présente une densité nettement plus forte $\left(\chi^{2}=6,8\right.$ comparé à $\left.\mathrm{B} 4\right)$. Pour les subadultes la seule différence significative est entre B5 et B6 $\left(\chi^{2}=4,6\right)$. Quelle que soit l'année ces différences se maintiennent, mais à l'intérieur d'une séquence il peut y avoir des variations interannées au niveau de la colonisation par chaque classe de taille (Tableau VIII). Pour B4 il y a une stabilité temporelle pour les immatures et les adultes, mais une variabilité significative pour les subadultes. Pour B5 et B6 seule la densité des subadultes est stable et pour B7 il n'y a pas de stabilité interannuelle quelle que soit la taille.

\section{Tableau V}

Incidence du type de séquences sur le niveau des captures en fonction de la taille des individus $\geq 1+$ dans le site aval.

\section{Table V}

Effect of sequence type on catches level in relation to individuals $\geq 1+$ size in downstream site.

\begin{tabular}{|c|c|c|c|c|}
\hline & \multicolumn{3}{|c|}{ Classes de taille $(\mathbf{m m})$} & \\
\hline Séquences & $\mathbf{2 0 - 4 0}$ & $\mathbf{4 1 - 6 0}$ & $\mathbf{+ 6 0}$ & Total \\
\hline $\mathrm{A} 1$ & 210 & 154 & 57 & 421 \\
\hline $\mathrm{A} 2$ & 174 & 133 & 70 & 377 \\
\hline $\mathrm{A} 3$ & 166 & 155 & 66 & 387 \\
\hline Total & 550 & 442 & 193 & 1185 \\
\hline
\end{tabular}




\section{Tableau VI}

Variations interannuelles des captures en fonction de la taille des individus $\geq 1+$ et du type de séquence dans le site aval (valeur limite du $\chi^{2}: 5,9$ ).

\section{Table VI}

Catches interannual variations in relation to individuals $\geq 1+$ size and sequence type in downstream site (threshold value of $\chi^{2}: 5.9$ ).

\begin{tabular}{|c|c|c|c|c|}
\hline & & \multicolumn{3}{|c|}{ Classes de taille $(\mathrm{mm})$} \\
\hline & & $20-40$ & $41-60$ & +60 \\
\hline \multirow{5}{*}{1991} & A1 & 19 & 39 & 8 \\
\hline & $\mathrm{A} 2$ & 32 & 31 & 5 \\
\hline & A3 & 28 & 67 & 16 \\
\hline & Total & 79 & 137 & 29 \\
\hline & $\chi^{2}$ & 3,4 & $5,8^{\star}$ & $6,3^{*}$ \\
\hline \multirow{5}{*}{1992} & $A 1$ & 54 & 20 & 7 \\
\hline & $A 2$ & 33 & 22 & 8 \\
\hline & A3 & 44 & 17 & 5 \\
\hline & Total & 131 & 59 & 20 \\
\hline & $\chi^{2}$ & 5,3 & 0,6 & 0,9 \\
\hline \multirow{5}{*}{1993} & A1 & 48 & 19 & 9 \\
\hline & $\mathrm{A} 2$ & 12 & 23 & 19 \\
\hline & A3 & 25 & 18 & 16 \\
\hline & Total & 85 & 60 & 44 \\
\hline & $\chi^{2}$ & $23,6^{\star}$ & 0,7 & 3,8 \\
\hline \multirow{5}{*}{1994} & A1 & 40 & 34 & 19 \\
\hline & $A 2$ & 34 & 16 & 23 \\
\hline & $A 3$ & 31 & 23 & 18 \\
\hline & Total & 105 & 73 & 60 \\
\hline & $\chi^{2}$ & 1,2 & $6,8^{*}$ & 0,7 \\
\hline \multirow{5}{*}{1995} & $A 1$ & 49 & 42 & 14 \\
\hline & $\mathrm{A} 2$ & 63 & 41 & 15 \\
\hline & A3 & 38 & 30 & 11 \\
\hline & Total & 150 & 113 & 40 \\
\hline & $\chi^{2}$ & $6,3^{\star}$ & 2,4 & 0,7 \\
\hline
\end{tabular}




\section{Tableau VII}

Incidence du type de séquence sur le niveau des captures en fonction de la taille des individus $\geq 1+$ dans le site amont ( $m$ : moyenne pour $10 \mathrm{~m}^{2}, \sigma$ : écart type, cv : coefficient de variation).

\section{Table VII}

Effect of sequence type on catches level in relation to individuals $\geq 1+$ size in upstream site ( $\mathrm{m}$ : average per $10 \mathrm{~m}^{2}, \sigma$ : standard deviation, cv : variation coefficient).

\begin{tabular}{|l|r|r|r|r|}
\hline \multirow{2}{*}{ Séquences } & \multicolumn{4}{|c|}{ Classes de taille (mm) } \\
\cline { 2 - 5 } & $\mathbf{2 0 - 4 0}$ & $\mathbf{4 1 - 6 0}$ & +60 & Total \\
\hline A - Captures totales & 20 & 17 & 16 & 53 \\
\hline B4 & 20 & 12 & 19 & 51 \\
\hline B5 & 209 & 103 & 39 & 351 \\
\hline B6 & 79 & 64 & 29 & 172 \\
\hline B7 & 328 & 196 & 103 & 627 \\
\hline Total & & & & \\
\hline B - Captures par 10 $\mathbf{m}^{\mathbf{2}}$ & 4,4 & 3,7 & 3,5 & 11,7 \\
\hline B4 & 2,5 & 1,5 & 2,3 & 6,3 \\
\hline B5 & 16,7 & 8,2 & 3,1 & 28,0 \\
\hline B6 & 3,9 & 3,2 & 1,4 & 8,6 \\
\hline B7 & 6,8 & 4,1 & 2,5 & 13,9 \\
\hline m & 6,6 & 2,8 & 0,9 & 9,8 \\
\hline$\sigma$ & 0,97 & 0,69 & 0,36 & 0,70 \\
\hline Cv & & & &
\end{tabular}

\section{Incidence de la variabilité structurelle des habitats}

Même si le site aval a présenté une certaine variabilité dans le niveau des captures au cours des 5 années, celle-ci ne peut être corrélée à des changements visibles de l'habitat. Ce n'est pas le cas du site amont où des variations notables ont été observées, au moins au niveau de certaines séquences.

\section{Barrage de branches sur B7 en 1991}

En 1991 les 3 à 4 mètres aval du sous secteur B7 étaient encombrés par un entrelacis de branchages d'un arbre abattu, avec accumulation de détritus (feuilles, branches) et ralentissement du courant. La surface concernée représentait $20 \%$ de B7 mais $61,1 \%$ des animaux capturés. Soit une densité de $27,5 / 10 \mathrm{~m}^{2}$ contre $4,3 / 10 \mathrm{~m}^{2}$ pour le reste de B7. Cette densité locale est alors proche de celle de B6 $\left(28,8 / 10 \mathrm{~m}^{2}\right)$. Autrement dit la complexification de l'espace dans cette zone de dépôt, augmente fortement la densité, par augmentation des abris, à un niveau comparable à celle des cailloux : la destruction volontaire du barrage à la fin 1991 provoque en 1992 un retour à une densité comparable à celle du reste de $B 7\left(4,7 / 10 \mathrm{~m}^{2}\right)$. 


\section{Tableau VIII}

Variations interannuelles des captures en fonction de la taille des individus $\geq 1+$ dans chaque type de séquence du site amont (valeur limite du $\chi^{2}: 9,5$ ).

\section{Table VIII}

Catches interannual variations in relation to individuals $\geq 1+$ size in each sequence type of upstream site (threshold value of $\chi^{2}: 9.5$ ).

\begin{tabular}{|c|c|c|c|c|}
\hline \multirow{2}{*}{\multicolumn{2}{|c|}{ Séquences }} & \multicolumn{3}{|c|}{ Classes de taille $(\mathrm{mm})$} \\
\hline & & \multirow{2}{*}{$\frac{20-40}{2}$} & \multirow{2}{*}{$\frac{41-60}{8}$} & \multirow{2}{*}{$\frac{+60}{4}$} \\
\hline \multirow{6}{*}{ B4 } & 1991 & & & \\
\hline & 1992 & 2 & 1 & 3 \\
\hline & 1993 & 5 & 1 & 3 \\
\hline & 1994 & 6 & 4 & 1 \\
\hline & 1995 & 6 & 4 & 6 \\
\hline & $x^{2}$ & 3,9 & $9,7^{*}$ & 2,4 \\
\hline \multirow{6}{*}{ B5 } & 1991 & 1 & 2 & 10 \\
\hline & 1992 & 0 & 1 & 0 \\
\hline & 1993 & 9 & 2 & 1 \\
\hline & 1994 & 7 & 3 & 4 \\
\hline & 1995 & 3 & 5 & 4 \\
\hline & $\chi^{2}$ & $14,9^{\star}$ & 3,5 & $16,2^{*}$ \\
\hline \multirow{6}{*}{ B6 } & 1991 & 45 & 35 & 15 \\
\hline & 1992 & 32 & 18 & 11 \\
\hline & 1993 & 65 & 18 & 8 \\
\hline & 1994 & 48 & 19 & 9 \\
\hline & 1995 & 19 & 23 & 2 \\
\hline & $\chi^{2}$ & $28,6^{\star}$ & 9,1 & $10,1^{*}$ \\
\hline \multirow{6}{*}{ B7 } & 1991 & 7 & 18 & 12 \\
\hline & 1992 & 7 & 10 & 2 \\
\hline & 1993 & 36 & 6 & 5 \\
\hline & 1994 & 26 & 16 & 5 \\
\hline & 1995 & 2 & 15 & 5 \\
\hline & $x^{2}$ & $54,6^{*}$ & $12,3^{*}$ & $9,4^{*}$ \\
\hline
\end{tabular}

\section{Accumulation de vase en aval du site $B$ en 1992}

En 1992 un amas de branches et détritus constituent un véritable barrage en aval du site, (à $4 \mathrm{~m}$ en aval de B4). Le ralentissement du courant et une augmentation du niveau de l'eau $(10-15 \mathrm{~cm})$ ont pour conséquence une sédimentation de fines particules visibles jusqu'en B6, ce barrage est détruit fin 1992.

Les captures sont réduites par 2,3 entre 1991 et 1992 au niveau de B4, par 13,5 pour B5, mais très peu $(1,5)$ pour B6. La récupération est nette en 1993 avec augmentation des captures dans les séquences envasées de 1992 (Tableau IX). Le profond limoneux de B5, normalement peu favorable, est le plus touché par l'envasement supplémentaire, les quelques abris (cailloux, feuilles mortes) ne sont plus accessibles. 


\section{Tableau IX}

Incidence de l'envasement de 1992 sur la densité des captures (pour $10 \mathrm{~m}^{2}$ ) dans les trois séquences de la partie aval du site $B$.

\section{Table IX}

Effect of silting during 1992 year on catches density (per $10 \mathrm{~m}^{2}$ ) in the three sequences of downstream part of site $B$.

\begin{tabular}{|c|c|c|c|}
\hline & B4 & B5 & B6 \\
\hline 1991 & 15,5 & 8,1 & 38,0 \\
\hline 1992 & 6,6 & 0,6 & 24,4 \\
\hline 1993 & 10 & 7,5 & 36,4 \\
\hline
\end{tabular}

Colmatage général du site $B$ en 1994-1995

En 1994 un colmatage est visible sur l'ensemble du site, plus faible que pour les séquences aval de 1992. II se remarque surtout par la mise en suspension de particules lors de prélèvements dans la zone B6 et par le colmatage des Fontinalis, non visible les autres années. Ce colmatage est encore observé en 1995, mais il est moins accentué. Compte tenu de sa régularité, il peut être mis en relation avec des travaux d'exploitation forestière en amont.

La réduction globale des adultes sur le site amont se remarque en particulier en 1995 alors que sur le site aval la population augmente (Tableaux II et III). La chute de densité concerne surtout la zone favorable B6 qui passe de 32 à 17,6 ind/10 $\mathrm{m}^{2}$ et B7 qui passe de 12,2 à $5,5 \mathrm{ind} / 10 \mathrm{~m}^{2}$. La réduction des juvéniles est sensible dès 1994 pour l'ensemble du site, avec une réduction drastique y compris dans B6 (Tableau III). Cette réduction des juvéniles de l'année 1994 se retrouve au niveau des immatures de 1995 surtout pour B6 et B7 (Tableau VIII). La réduction entre 1993 et 1994 étant moins forte, l'effet du colmatage partiel du site s'accentue avec le temps.

\section{sites}

Stabilité et variabilité dans la sélection des mésohabitats sur l'ensemble des

L'application du facteur de correction d'efficacité $(1,56)$ sur les données (individus $\geq 1+$ ) du site aval, pour des comparaisons avec le site amont, ne change pas la hiérarchie des valeurs, l'aval ayant les densités les plus élevées même sans correction.

\section{Variabilité du peuplement en fonction des séquences des deux sites}

La comparaison à l'aide du coefficient de Spearman de la hiérarchie des densités de capture entre les séquences montre :

- au niveau des individus $\geq 1+$ : l'ordre du choix des différentes séquences ne change pas au cours des années (Tableau X A). L'année 1993 montre une tendance à être un peu différente en particulier vis-à-vis de 1991 et 1995. Par contre en comparant dans chaque séquence les changements d'abondance au cours de chaque année, seul B5 et B7 sont corrélées significativement (Tableau XI A). Autrement dit ces deux profonds présentent la même attraction pour les animaux d'année en année, proportionnellement à l'évolution. II semble exister quelques tendances inversées entre B6 et le site aval, ou encore des effets de proximité dans la mesure où 2 séquences adjacentes présentent globalement des relations plus fortes. 
Tableau X

Corrélations entre les densités des captures de chaque année dans l'ensemble des séquences (valeur limite du coefficient de Spearman : 0,74).

Table X

Correlations between catches densities of each year in whole sequences (threshold value of Spearman coefficient : 0.74 ).

\begin{tabular}{|l|c|c|c|c|c|}
\hline & 1991 & 1992 & 1993 & 1994 & 1995 \\
\hline \multicolumn{1}{|l|}{ A-Individus $\geq 1+$} & 0,85 & 0,78 & 0,89 & 0,89 \\
\hline 1991 & 1 & 1 & 0,96 & 0,96 & 0,86 \\
\hline 1992 & & & 1 & 0,89 & 0,78 \\
\hline 1993 & & & & 1 & 0,89 \\
\hline 1994 & & & & & 1 \\
\hline 1995 & & & $-0,03$ & 0,67 & 0,92 \\
\hline B-Juvéniles & 1 & 0,60 & 0,40 & 0,39 & 0,78 \\
\hline 1991 & 1 & 1 & $-0,14$ & 0,14 \\
\hline 1992 & & & & 1 & 0,85 \\
\hline 1993 & & & & & 1 \\
\hline 1994 & & & & & \\
\hline 1995 & & & & & \\
\hline
\end{tabular}

Tableau XI

Corrélations entre les densités des captures dans chaque séquence sur l'ensemble des années (valeur limite du coefficient de Spearman : 0,90).

Table XI

Correlations between catches densities in each sequence of whole years (threshold value of Spearman coefficient : 0.90 ).

\begin{tabular}{|l|c|c|c|c|c|c|c|}
\hline & A1 & A2 & A3 & B4 & B5 & B6 & B7 \\
\hline \multicolumn{7}{|l|}{ A - Individus $\geq 1+$} \\
\hline A1 & 1 & 0,7 & 0 & 0,3 & $-0,2$ & $-0,6$ & $-0,1$ \\
\hline A2 & & 1 & 0,7 & 0,8 & 0,2 & $-0,6$ & 0 \\
\hline A3 & & & 1 & 0,8 & 0,3 & $-0,5$ & $-0,1$ \\
\hline B4 & & & & 1 & 0,3 & $-0,4$ & 0,1 \\
\hline B5 & & & & & 1 & 0,6 & 0,9 \\
\hline B6 & & & & & & 1 & 0,8 \\
\hline B7 & & & & & & & 1 \\
\hline B - Juvéniles & & 0,2 & 0 & 0,2 & 0,5 & 0,5 & 0,7 \\
\hline A1 & 1 & 1 & 0,9 & $-0,7$ & $-0,6$ & $-0,6$ & $-0,2$ \\
\hline A2 & & & 1 & $-0,6$ & $-0,7$ & $-0,7$ & $-0,4$ \\
\hline A3 & & & & 1 & 0,9 & 0,9 & 0,7 \\
\hline B4 & & & & & 1 & 1 & 0,9 \\
\hline B5 & & & & & & 1 & 0,9 \\
\hline B6 & & & & & & & 1 \\
\hline B7 & & & & & & & \\
\hline
\end{tabular}


- au niveau des juvéniles : la variabilité est plus forte, seule l'année 1995 montre un choix comparable à 1991, 1992 et 1994, toutes les autres années diffèrent entre elles. L'année 1993 est la plus originale en relation avec une forte densité en amont par rapport à l'aval (Tableau $X \mathrm{~B}$ ). Les changements interannuels dans chacun des habitats sont souvent semblables (Tableau XI B). Ainsi $A 2$ et $A 3$ sont liés de même que $B 4$ évolue comme B5 et B6, B5 comme B6 et B7, B6 comme B7. Si globalement l'évolution du peuplement des séquences de l'aval est indépendante de celle de l'amont (avec même des tendances opposées), il existe aussi quelques différences intra site (A1 versus A2 et A3).

\section{Relations entre la densité du peuplement et la nature du substrat}

La comparaison entre les fréquences de recouvrement de chaque faciès par les principaux substrats (Tableau I) et l'abondance des captures permet d'analyser les préférences de l'espèce, en particulier en fonction de la taille des individus (Tableau XII).

La densité en juvéniles est corrélée à l'importance des zones de cailloux, il en est de même pour les immatures, dans une moindre mesure pour les subadultes. La corrélation n'est pas significative pour les adultes. Les substrats caillouteux sont donc choisis d'autant plus que la taille des individus est faible. Les zones de graviers laissent tous les stades indifférents. Les substrats sableux sont évités par tous les individus et ce d'autant plus significativement qu'ils sont petits.

Cette préférence pour les zones de cailloux pour la majorité des individus peut être analysée plus finement en relation avec la densité des gros cailloux (Tableau I). Quelle que soit la taille individuelle, la densité des captures est corrélée fortement à la densité des gros cailloux (Tableau XII). Des relations linéaires peuvent être calculées entre cette densité $(C)$ et celle des différentes classes de taille sur l'ensemble des années :

$\begin{array}{llr}\text { d (Juvéniles) } & = & 4,37 C+1,27(r=0,96) ; \\ d \text { (individus } \geq 1+) & = & 19,25 C-4,35(r=0,93) ; \\ d(20-40) & = & 9,45 C-2,64(r=0,96) ; \\ d(41-60) & = & 7,28 C-2,05(r=0,91) \\ d(+60) & = & 2,65 C+0,06(r=0,84)\end{array}$

Tableau XII

Corrélations entre les densités des captures, la taille des individus et les principales caractéristiques du substrat (valeur limite du coefficient de Spearman : 0,74).

Table XII

Correlations between catches densities, individual size and main characteristics of substratum (threshold value of Spearman coefficient : 0.74 ).

\begin{tabular}{|l|c|c|c|c|}
\hline Substrats & Juvéniles & Immatures & Subadultes & Adultes \\
\hline Cailloux & 1 & 0,96 & 0,78 & 0,71 \\
\hline Graviers & 0,11 & 0,08 & 0,32 & 0,08 \\
\hline Sables & $-0,96$ & $-0,89$ & $-0,75$ & $-0,57$ \\
\hline Densité des cailloux de $+20 \mathrm{~cm}$ & 0,97 & 1 & 0,90 & 0,82 \\
\hline
\end{tabular}


Ce choix des zones caillouteuses reste stable dans le temps. Pour l'ensemble des individus autres que les juvéniles, la corrélation (de Spearman) entre la densité des gros cailloux et celle des écrevisses reste significative au cours des 5 années (1991 Rs = 0,92 ; $1992 \mathrm{Rs}=0,82 ; 1993 \mathrm{Rs}=0,86 ; 1994 \mathrm{Rs}=0,92 ; 1995 \mathrm{Rs}=0,92)$. Pour les juvéniles si les années 1991, 1992, 1994 et 1995 montrent une relation significative entre l'abondance des individus et la densité des gros cailloux ( $R s=0,81-0,78-0,89-0,96$ respectivement) ce n'est pas le cas pour 1993 (Rs =0,18). Pour cette année les résultats sont affectés par la faiblesse des captures en aval contrairement à l'amont.

\section{Relation entre les densités de capture des diverses classes de taille}

La tendance générale des différentes classes de taille est de choisir les mêmes faciès, en particulier les zones de courant avec des cailloux. Le choix des individus d'une classe de taille entre les différentes séquences doit donc être comparable à celui d'une autre classe de taille. C'est effectivement le cas général, les corrélations sont d'autant plus fortes que les tailles sont proches, c'est-à-dire que les besoins individuels sont comparables. Cependant les classes de taille les plus opposées, juvéniles versus adultes de $+60 \mathrm{~mm}$, sont à la limite du seuil de signification à $5 \%$, elles tendent donc à différer dans leur choix (Tableau XIII).

En considérant le peuplement globalement stable à un instant donné, la quantité de juvéniles doit être proportionnelle au nombre d'adultes présents, c'est-à-dire leurs géniteurs. Les densités sur $10 \mathrm{~m}^{2}$ sont reliées:

df = densité $/ 10 \mathrm{~m}^{2}$ des adultes $+60 \mathrm{~mm}$;

$\mathrm{d}($ Juv $)=2,90 \mathrm{df}^{0,705}$ avec $\mathrm{r}=0,81$ pour $\mathrm{n}=7$ (valeur limite à $5 \%, r=0,67$ ).

\section{Tableau XIII}

Corrélations entre les densités des captures dans chaque classe de taille (valeur critique du coefficient de Spearman : 0,74).

\section{Table XIII}

Correlations between catches densities in each size class (threshold value of Spearman coefficient : 0.74 ).

\begin{tabular}{|l|c|c|c|c|}
\hline & Juvéniles & Immatures & Subadultes & Adultes \\
\hline Juvéniles & 1 & 0,97 & 0,80 & 0,72 \\
\hline Immatures & & 1 & 0,90 & 0,82 \\
\hline Subadultes & & & 1 & 0,82 \\
\hline Adultes & & & & 1 \\
\hline
\end{tabular}

\section{DISCUSSION ET CONCLUSION}

Au cours d'une étude préliminaire, les peuplements les plus denses de l'écrevisse à pattes blanches ont été trouvés dans les zones de courant de différents ruisseaux de Normandie. Le choix de cet habitat était particulièrement accentué au niveau des juvéniles, plus qu'au niveau des adultes récoltés aussi dans certaines zones à faible courant, avec ombrage ou non (NEVEU, 1996). 
Dans une nouvelle étude focalisée sur un seul ruisseau, mais avec un suivi sur 5 années successives, l'analyse de la répartition transversale des individus a pu être précisée avec une caractérisation des divers mésohabitats. Chacun de ces mésohabitats a été échantillonné séparément sur deux sites, en aval et en amont du ruisseau, au mois d'octobre de chaque année pour être dans des conditions comparables par rapport au cycle de développement de l'espèce. Cette période correspond au début des accouplements et pourrait être considérée a priori comme particulière par rapport à la répartition spatiale des sexes et des tailles. Mais des échantillons complémentaires effectués dans le site aval en juin, août 1992 et juin 1993 ne montrent pas de différence quant au choix des trois séquences par les individus $\geq 1+$ (juin $1992: \chi^{2}=5,5$; août 1992 : $\chi^{2}=3,1$; juin $1993: \chi^{2}=3,8$ ). De même la partie A1 reste souvent la plus peuplée avec respectivement $41 \%, 33 \%, 43 \%$ des captures du site et le sex-ratio reste stable (respectivement $0,43-0,50-0,43$ ).

Pour les juvéniles les comparaisons intersaisons sont plus difficiles en rapport avec une croissance rapide la 1 ère année et leur capturabilité très faible en juillet-août corrélée à leur très faible taille (10 à $12 \mathrm{~mm}$ ) (NEVEU, 2000).

En aval du ruisseau le peuplement peut varier d'une année à l'autre, mais reste relativement stable au niveau de la répartition interséquences pour les individus de plus d'un an, en liaison avec un mésohabitat assez homogène. Par contre au niveau des juvéniles, il y a un gradient de l'aval vers l'amont du site, la partie la plus en aval étant la plus peuplée, avec une plus grande variabilité temporelle.

Dans le site amont le contraste interfaciès est plus fort, l'hétérogénéité du peuplement aussi. Les zones de cailloux sont les plus peuplées et s'opposent aux profonds limoneux moins peuplés. Les juvéniles se trouvent présents dans tous les habitats, mais sont surtout abondants dans les zones courantes. L'observation directe lors des relevés de chaque épuisette montre qu'ils ne sont pas spécialement présents dans les touffes de Fontinalis, comme dans le cas de l'étude de BROWN et BOWLER (1977). II faut cependant noter que dans ce travail, la structure relativement monotone du substrat peut avoir orienté plus fortement les jeunes écrevisses vers les Fontinalis en tant que zone refuge. Ce phénomène est particulièrement net en étang pour plusieurs espèces où la rareté des abris augmente fortement l'attractivité d'un herbier isolé, comparativement à un étang riche en macrophytes (obs. pers.). Ce rôle de zone refuge peut se retrouver au niveau des radicelles issues des arbres des berges. Au cours des prélèvements, l'observation directe ne montre pas une localisation particulière des animaux dans ce substrat (absent du site $B$, limité dans le site $A$ à la mouille de concavité de $A 3$ et au chenal lotique de A2), même au niveau des juvéniles contrairement à certaines observations (FOSTER, 1995 ; SMITH et al., 1996). Au moment des mues ce substrat pourrait paraître plus sécurisant, or toutes les mues récoltées au cours des pêches étaient coincées entre les cailloux. En étang il est courant de retrouver de nombreuses mues accrochées dans les herbiers lorsque ceux-ci sont isolés (effet refuge), dans le cas contraire les mues sont dispersées (obs. pers.) Par contre la variabilité des captures de juvéniles est élevée, ce qui peut être lié à des variations de production de la part des géniteurs, mais aussi à une certaine hétérogénéité de la capturabilité d'une année à l'autre.

Le sex-ratio est stable dans chacun des mésohabitats, il peut varier légèrement au cours des années en particulier dans le site aval. L'habitat des deux sexes est donc comparable à cette période de l'année proche de la reproduction.

Dans la plupart des séquences les rapports entre les densités des différentes classes de taille restent a priori corrélés aux mortalités liées à l'âge. II peut y avoir quelques variations dans la microrépartition des classes de taille, surtout en amont, mais les plus grands individus ont la répartition la plus homogène. ROQUEPLO (1997) trouve par contre un maximum de gros individus dans les profonds. 
Des changements momentanés de la structure de l'habitat peuvent influencer la densité du peuplement. Ainsi l'accumulation de débris végétaux (branches, feuilles) augmente d'une part le nombre d'abris disponibles, mais d'autre part provoque un envasement par effet barrage. Dans le $1^{\text {er }}$ cas la densité augmente fortement et atteint celle des zones de cailloux, à condition que les débris soient bien percolés par un courant d'eau. Dans le $2^{\text {ème }}$ cas la densité est réduite sous l'effet du colmatage auquel les jeunes individus sont plus sensibles (difficultés de déplacement, besoins élevés en oxygène). L'évitement des zones vaseuses permanentes a été remarqué par plusieurs auteurs (HOGGER, 1988 ; SUMMERS, 1996 in NAURA et ROBINSON), mais une certaine tolérance est aussi observée (JAY et HOLDICH, 1981 ; NAURA et ROBINSON, 1998). Par contre l'accumulation de tas de feuilles mortes peut aussi servir de nourriture (KAUSHIK et BIRD, 1987 ; FOSTER, 1995).

Dans le cas des animaux de plus d'un an, la hiérarchie dans le choix des habitats ne change pas et la relation entre la densité du peuplement de deux séquences est d'autant plus forte que celles-ci sont contiguës. Pour les juvéniles les variations dans les choix sont plus fortes. Par ailleurs les variations du peuplement des séquences aval sont indépendantes de celles des séquences amont, avec même une tendance inverse dans leur évolution.

Il existe une relation entre l'abondance des cailloux et celle des écrevisses, celle-ci est d'autant plus forte que les individus sont jeunes. Cette liaison est particulièrement significative au niveau des gros cailloux (supérieurs à $20 \mathrm{~cm}$ ) et reste stable au cours des années. Ce choix des zones caillouteuses a été remarqué par plusieurs auteurs (BROWN et BOWLER, 1977 ; NAURA et ROBINSON, 1998), il est plus accentué que pour Astacus astacus (LACHAT et LAURENT, 1988). Ce comportement peut s'expliquer *par la recherche d'abris de taille suffisante, ainsi FOSTER (1993) montre une relation entre la surface des cailloux (et pas leur épaisseur) et la taille des individus. Cependant ce n'est pas le cas ici où toutes les tailles se trouvent dans les mêmes séquences de cailloux. La stabilité des gros cailloux peut aussi être une garantie pour la production régulière de périphyton (FOSTER, 1995) ou le support d'une faune riche en macroinvertébrés (NEVEU et al., 1979) appréciée par les écrevisses (GODDARD, 1988 ; ZEKHNINI et CHAISEMARTIN, 1991). Dans les deux cas les zones de cailloux offrent une sécurité alimentaire.

Les zones de graviers laissent les écrevisses du ruisseau indifférentes, alors que ROQUEPLO (1997) trouve le maximum d'individus dans ce substrat. Les faciès sablo-limoneux sont peu appréciés, d'autant plus que les individus sont jeunes. II est possible de relier ce comportement au risque de prédation par les poissons dans les profonds, mais aussi à un habitat moins oxygéné, plus instable pour la marche et plus pauvre en nourriture (macroinvertébrés).

Plusieurs auteurs signalent le rôle de certains substrats comme support de juvéniles : litière, feuilles, radicelles, ... (ARRIGNON et ROCHE, 1981 ; DAGUERRE de HUREAU et ROQUEPLO, 1981 ; FOSTER, 1995). Ce n'est pas le cas dans cette étude et il en est de même de certaines zones, telles que les creusements dans les berges meubles favorables aux adultes (BROWN et BOWLER, 1977 ; SMITH et al., 1996), du reste très rares ici.

L'influence de l'ombrage est difficile à évaluer, les deux sites étant sous couvert forestier. Mais plusieurs études montrent que les écrevisses choisissent ces zones (SMITH et al., 1996 ; NAURA et ROBINSON, 1998). Dans la pré-étude régionale menée sur divers ruisseaux de Normandie, ce facteur ne semblait pas être très ségrégatif (NEVEU, 1996).

Compte tenu d'une tendance générale pour toutes les tailles à se trouver dans les mêmes substrats, il est possible de trouver des relations étroites entre la densité d'une classe d'âge et celle d'une autre classe. Cette relation est d'autant plus forte que les 
individus sont proches en taille, c'est-à-dire ont des besoins fonctionnels comparables. Par contre les tailles les plus opposées présentent quelques divergences, c'est le cas des juvéniles par rapport aux grands adultes, ce qui permet d'éviter entre autres une trop grande prédation de ces derniers.

II est possible de s'interroger sur la relative homogénéité des choix par les divers stades, qui a priori pourrait augmenter la compétition entre les différents âges et sexes. Mais le choix préférentiel des substrats les plus complexes au niveau spatial permet de réduire les contacts interindividuels, tout en favorisant la diversité des ressources trophiques (périphyton, détritus, macroinvertébrés). Il reste à vérifier s'il y a un partage de ces ressources, sachant que globalement les jeunes stades sont plutôt plus carnassiers que les plus âgés. Ces choix sont aussi la résultante des besoins métaboliques de cette espèce, avec un taux d'oxygène garanti par la vitesse du courant élevée des faciès caillouteux. Par ailleurs, la structure du substrat et sa stabilité peuvent favoriser un ancrage plus aisé pour la locomotion et offrir des abris pour cette espèce essentiellement lucifuge, comparativement à une espèce pluriactive comme Procambarus clarkii qui recherche peu les abris ombragés (obs. pers.).

Ces résultats permettent d'envisager des aménagements du substrat pour augmenter la densité dans les zones faiblement peuplées avec pour principe de base d'augmenter les abris en complexifiant l'espace accessible aux écrevisses.

L'impact de l'envasement est un bon indicateur des risques liés à une reprise d'une certaine érosion et à l'influence des travaux forestiers menés sur des parcelles de grandes surfaces et en pente. De même l'abandon de branches, voir de troncs d'arbres, provoque des barrages qui en ralentissant le courant permettent aux fines de se déposer et de colmater les substrats favorables aux écrevisses. Des mesures simples au niveau des activités forestières peuvent ainsi être dictées par ces observations de façon à protéger le ruisseau de ces impacts.

Les premiers résultats sur ces deux sites d'étude montrent des différences au niveau de la croissance. Les individus du site amont ont une croissance plus rapide, mais plus homogène dans le temps que ceux du site aval (NEVEU, 2000).

Ces nouveaux résultats sur l'occupation de l'espace montrent que les densités de population des différents habitats de l'aval sont plus élevées qu'en amont, mais que l'ordre de préférence des séquences reste stable, même si les densités sont variables d'une année à l'autre. Malgré un substrat favorable dominant et homogène dans les différentes séquences de l'aval, la forte densité peut être un des facteurs qui freine la croissance. En amont au contraire des substrats plus contrastés et une certaine instabilité (embâcles) n'optimise pas la densité et réduit a priori la compétition en faveur d'une croissance meilleure, liée par ailleurs à des conditions thermiques plus élevées de 1 à $3^{\circ} \mathrm{C}$ pour les maxima estivaux (NEVEU, op. cit.).

L'ensemble des connaissances acquises sur le ruisseau du Vivier montre comment une population d'écrevisses à pattes blanches, globalement stable à l'échelle de 5 années grâce a priori à un environnement protecteur (la forêt), présente cependant une variabilité locale. Cette variabilité, tant au niveau de la croissance que de la densité inter-habitats, est liée à de petites variations du milieu aquatique, quelles soient thermiques, granulométriques ou structurelles (abris), ayant une influence locale ; ainsi l'abondance des juvéniles en aval est indépendante de celle de l'amont. Cette association entre stabilité et variabilité peut être une garantie de la pérennité de la population en dehors de toute intervention d'un éventuel facteur catastrophique détruisant tout (pathologie) ou partie du stock (curage, colmatage, ...). 


\section{BIBLIOGRAPHIE}

ARRIGNON J., ROCHE B., 1981. Population of the crayfish Austropotamobius pallipes pallipes Lereb. in a brook of Corsica, France. Freshwater Crayfish, V, 229-238.

BIKUNA B.G., DOCAMPO L., ASENSIO R., 1989. Distribution et autoécologie de l'écrevisse à pattes blanches Austropotamobius pallipes (Ler.) à Bizkaia (Pays Basque, Espagne). Ann/s Limnol., 25, 219-229.

BOHL E., 1997. An isolated population of the white-clawed crayfish (Austropotamobius pallipes) in the principality of Liechtenstein. Bull. Fr. Pêche Piscic., 347, 701-712.

BROWN D.J., BOWLER K., 1977. A population study of the British freshwater crayfish Austropotamobius pallipes (Lereboullet). Freshwater crayfish, III, 33-49.

DAGUERRE DE HUREAUX N., ROQUEPLO C., 1981. Définition du biotope préférentiel de l'écrevisse à pattes blanches Austropotamobius pallipes dans un ruisseau landais. Bull. Fr. Pêche Piscic., 281, 211-222.

FOSTER J., 1993. The relationship between refuge size and body size in the crayfish Austropotamobius pallipes (Lereboullet). Freshwater Crayfish, IX, 245-249.

FOSTER J., 1995. Factors influencing the distribution and abundance of the crayfish Austropotamobius pallipes (Lereboullet) in Wales and the Marches, UK. Freshwater Crayfish, VIII, 78-98.

GHERARDI F., BARBARESI S., VILLANELLI F., 1998. Movement patterns of the white-clawed crayfish Austropotamobius pallipes, in a Tuscan Stream. J. Freshw. Ecol., 13, 413-423.

GODDARD J.S., 1988. Food and feeding. In : HOLDICH D.M. and LOWERY R.S. (Eds). Freshwater Crayfish : Biology, Management and Exploitation. Croom Helm, London, $498 \mathrm{p}$.

HOGGER J.B., 1988. Ecology, population biology and behaviour. In : HOLDICH D.M. and LOWERY R.S. (Eds). Freshwater Crayfish : Biology, Management and Exploitation, Croom Helm, London, 498 p.

JAY D., HOLDICH D.M., 1981. The distribution of the crayfish Austropotamobius pallipes in British waters. Freshwater Biology, 11, 121-129.

KAUSHIK N.K., BIRD G.A., 1987. Processing of maple, leaf, grass and fern packs and their colonisation by invertebrates in a stream. J. Freshw. Ecol., 4, 177-189.

LACHAT G., LAURENT P.J., 1988. The habitats of Astacus astacus L. and Austropotamobius pallipes Ler. in the Morvan. Freshwater Crayfish, VII, 61-68.

LEVEQUE C., 1995. L'habitat : être au bon endroit au bon moment? Bull. Fr. Pêche Piscic., 337-338-339, 9-20.

MALAVOI J.R., 1989. Typologie des faciès d'écoulement ou unités morphodynamiques des cours d'eau à haute énergie. Bull. Fr. Pêche Piscic., 315, 189-210.

NAURA M., ROBINSON M., 1998. Principles of using river habitat survey to predict the distribution of aquatic species : an example applied to the native white clawed crayfish Austropotamobius pallipes. Aquatic Conserv. Mar. Freshw. Ecosyst., 8, 515-527.

NEVEU A., LAPCHIN L., VIGNES J.C., 1979. Le macrobenthos de la basse Nivelle, petit fleuve côtier des Pyrénées-Atlantiques. Ann. Zool. Ecol. Anim., 11, 85-111.

NEVEU A., 1996. Caractéristiques démographiques de stocks résiduels de l'écrevisse à pattes blanches, Austropotamobius pallipes (Astacidae) en Normandie. Cybium, 20, suppl., 75-93. 
NEVEU A., 2000. Etude des populations d'Austropotamobius pallipes (Crustacea, Astacidae) dans un ruisseau forestier de Normandie. I. Structures démographiques et croissance : stabilité et variabilité au cours de six années. Bull. Fr. Pêche Piscic., 356, 71-98.

ORTH D.J., 1995. Influence du compartiment trophique dans les réponses des populations de poissons aux variations artificielles de débit. Bull. Fr. Pêche Piscic., 337-338-339, 317-328.

ROQUEPLO C., 1997. Etude de populations d'écrevisses à pattes blanches (Austropotamobius pallipes, Lereboullet) en cours d'eau soumises à une pêche de loisir. L'Astaciculteur de France, 51, 59-70.

SCHERRER B., 1984. Biostatistique. MORIN (ed.), Boucherville, Québec, 850 p.

SMITH G.R.T., LEARNER M.A., SLATER F.M., FOSTER J., 1996. Habitats features important for the conservation of the native crayfish, Austropotamobius pallipes in Britain. Biol. conserv., 75, 239-246.

SUMMERS D.M., 1996. A preliminary investigation into the distribution and habitat use of white-clawed crayfish (Austropotamobius pallipes) in the River Piddle. The game Conservary Trust, Hampshire.

ZEKHNINI A., CHAISEMARTIN C., 1991. Structure démographique et éthologie alimentaire de l'écrevisse pallipède (Austropotamobius pallipes) dans quatre cours d'eau. Vie Milieu, 41, 45-53. 